
\title{
3 Research Square \\ The effects of type 2 diabetes mellitus on the corneal endothelium and central corneal thickness
}

Tae Gi Kim ( $\nabla$ tk1213@hanmail.net )

Kyung Hee University Hospital at Gangdong

Yoo Jin Kim

Kyung Hee University Hospital at Gangdong

\section{Research Article}

Keywords: corneal endothelial cell morphology, type 2 diabetes, central corneal thickness

Posted Date: December 9th, 2020

DOI: https://doi.org/10.21203/rs.3.rs-109433/v1

License: (c) (1) This work is licensed under a Creative Commons Attribution 4.0 International License. Read Full License

Version of Record: A version of this preprint was published at Scientific Reports on April 15th, 2021. See the published version at https://doi.org/10.1038/s41598-021-87896-3. 


\section{Abstract}

Aim of this study is to evaluate the differences in corneal endothelial cell morphology and corneal thickness in patients with and without type 2 diabetes related to age, disease duration, and HbA1c percentage. This retrospective cross-sectional study included 511 type 2 diabetes patients (1022 eyes) and 900 (1799 eyes) non-diabetic patients. The endothelial cell density (ECD), variation in endothelial cell size (CV), percentage of hexagonal cells, and central corneal thickness (CCT) were analyzed using a noncontact specular microscope and a Pentacam Scheimpflug camera. We also examined the correlation between the corneal parameters and the duration of diabetes. Blood $\mathrm{HbA} 1 \mathrm{c}$ results in $\mathrm{DM}$ patients were used within 2 months of ophthalmic examination. The controls had no diabetes confirmed by blood tests. For all ages, the subjects with type 2 diabetes showed significantly lower ECD, hexagonality, higher CV, and thicker CCT than the control group. This difference was more pronounced in patients with longstanding DM ( $\geq 10$ years) and high HbA1c ( $\geq 7 \%$ ). When stratified by age group, from the 60 s group, corneal endothelial cell parameters showed a statistically significant difference between DM and control groups. The duration of diabetes was inversely correlated with ECD ( $r=-0.167 ; \mathrm{P}=0.000)$, but HbA1c was not correlated with ECD. These findings suggest that diabetes affects corneal endothelial cell in older age and those with long-standing DM and higher HbA1c. Regular corneal endothelial examinations are required in diabetic patients.

\section{Introduction}

The corneal endothelium is a single layer of cells that plays a major role in maintaining the optical transparency of the cornea through the Nap/Kp-ATPase pump activity. ${ }^{1}$ Corneal endothelial cells have the highest density at birth, which decreases by approximately $0.5 \%$ per year following aging. ${ }^{2}$ Factors other than aging that affect corneal endothelial cell loss include genetics, race, trauma, intraocular surgery, and infection. ${ }^{3-8}$

Diabetes mellitus (DM) is a metabolic disease characterized by chronic hyperglycemia resulting from dysfunctional insulin secretion or insulin action. Chronic hyperglycemia may lead to micro- and macrovascular disorders, and it causes changes in almost all ocular structures of the eye including changes in corneal endothelial cell density (ECD). Diabetes may lead to micro- and macrovascular complications, which may result in ocular manifestations such as diabetic retinopathy. Diabetic retinopathy is the most common ophthalmic complication of diabetes, but diabetes can also affect the cornea. ${ }^{9}$ Since the cornea is avascular, it is unlikely that vascular complications of DM will occur, but there is extensive literature on the association between diabetes and corneal endothelial cells. ${ }^{10,11}$

The conclusions of previous studies on the effect of DM on the corneal endothelium are inconsistent. In previous studies that compared type 1 and type 2 DM patients with normal subjects, ECD was reported to decrease in both DM group. ${ }^{12}$ However, some studies reported that there was no difference between type 2 DM patients and normal subjects. ${ }^{13}$ Lee et al. ${ }^{14}$ reported that patients with diabetes that had lasted for 10 years had more corneal morphological abnormalities regardless of the type of diabetes. On the other 
hand, Choo et al. ${ }^{11}$ reported that ECD decreased in diabetes, but they did not show any difference in coefficient of variation (CV), hexagonality, and central corneal thickness (CCT) in type 2 DM. StorrPaulsen et al. ${ }^{15}$ reported that CCT was significantly increased in the diabetic group, and higher HbA1c was associated with lower ECD in type 2 DM.

As indicated above, the changes in corneal endothelial cells in type $2 \mathrm{DM}$ are still controversial different from those of type 1 DM. However, most of the studies used small samples, and those that used agebased analysis are rare. Rachapalle et al. ${ }^{10}$ compared 1191 diabetic patients stratified by age and controls, only 121 normal subjects were included in the study. Su et al. ${ }^{16}$ analyzed 3239 people and a larger sample than this study, but they did not stratify them by age group for analysis.

The aim of the present study was to compare the corneal endothelial cell characteristics, such as ECD, CV, hexagonality, and CCT, of type 2 diabetic patients and healthy controls stratified by age group. In addition, the correlations between the duration of diabetes, $\mathrm{HbA} 1 \mathrm{c}$, and corneal endothelial cell morphology were also analyzed.

\section{Results}

A total of 1411 patients were included in the study: 1022 eyes in 511 patients (264 males, 247 females, mean age $65.6 \pm 11.1$ years) were included in the DM group, and 1799 eyes in 900 patients (333 males, 567 females, mean age $67.9 \pm 11.2$ years) were included in the control group. All patients were Asians. The mean duration of the DM was $10.8 \pm 8.7$ years, and the mean level of HbA1c level was $7.54 \pm 1.78 \%$ in the DM group. There were no differences between the groups in terms of age. The demographics and age distribution of the DM and control groups are shown in Table 1. 
Table 1

Demographics and age distributions of diabetic and control groups

\begin{tabular}{|llll|}
\hline & DM group & Control Group & *p value \\
\hline Sample size (Eyes) & $511(1022)$ & $900(1799)$ & - \\
\hline Sex (M:F) & $264: 247(528:$ & $333: 567(666:$ & \\
\hline Age, yr (Mean \pm SD) & $494)$ & $1133)$ & 0.637 \\
\hline HbA1c (Mean \pm SD) & $65.6 \pm 11.1$ & $67.9 \pm 11.2$ & - \\
\hline $\begin{array}{l}\text { Duration of Diabetes (Years) (Mean } \pm \\
\text { SD) }\end{array}$ & $7.54 \pm 1.78$ & - & - \\
\hline Age distribution (Eyes) & $10.8 \pm 8.7$ & - & - \\
\hline $40-49$ & & & - \\
\hline $50-59$ & 92 & 115 & - \\
\hline $60-69$ & 163 & 182 & - \\
\hline $70-79$ & 378 & 650 & \\
\hline$>80$ & 302 & 606 & \\
\hline p value by independent t-test & 87 & 246 & \\
\hline
\end{tabular}

\section{Comparative analysis of corneal parameters in all age group}

For all ages, the corneal parameters in the DM and control groups were significantly different. (Table 2). The ECD and hexagonality were significantly lower in the DM group than in the control group $(p=0.023$ and 0.030 , respectively), and CV and CCT were significantly greater in the DM group $(p=0.000$ and 0.000 , respectively).

Table 2

Comparison of the mean values of ECD, CV, hexagonality, and CCT of diabetic eyes and controls across all ages (mean \pm SD)

\begin{tabular}{|lllc|}
\hline & DM group & Control Group & *p \\
\hline ECD, cells $/ \mathrm{mm}^{2}$ & $2656.42 \pm 379.21$ & $2688.77 \pm 351.96$ & 0.023 \\
\hline Coefficient of Variation (\%) & $36.09 \pm 8.00$ & $34.92 \pm 7.16$ & 0.000 \\
\hline Hexagonal cell ratio (\%) & $53.55 \pm 9.80$ & $54.41 \pm 10.39$ & 0.030 \\
\hline CCT $(\mu \mathrm{m})$ & $551.80 \pm 34.10$ & $542.63 \pm 33.79$ & 0.000 \\
\hline $\begin{array}{l}\text { ECD: } \text { Endothelial Cell Density; CV: Coefficient of Variation; CCT: Central Corneal Thickness. *P-value } \\
\text { by independent t-test }\end{array}$ & & \\
\hline
\end{tabular}


When analyzed according to the duration of diabetes, the ECD, CV, hexagonality, and CCT of the group of patients with DM lasting 10 years or more were significantly different from those of the control group (Fig. 1A - D). However, in the group of patients with DM lasting for less than 10 years, only CV and CCT were significantly different from those of the control group. Only ECD was significantly different in the long duration ( $\geq 10$ years) and short duration (<10 years) groups.

When analyzed according to $\mathrm{HbA1c}$, in high $\mathrm{HbA1c}$ group ( $\geq 7 \%$ ), $\mathrm{ECD}, \mathrm{CV}$, and CCT were statistically significantly different compared to the control groups. Meanwhile, in the group with $\mathrm{HbA} 1 \mathrm{c}<7 \%$, only $\mathrm{CV}$ and CCT were significantly different (Fig. 1E - H). There was no statistically significant difference between the groups with $\mathrm{HbA} 1 \mathrm{c}$ of $\geq 7$ and $<7 \%$.

\section{Comparative analysis of corneal parameters by age group}

ECD, CV, and hexagonality of the DM and control groups stratified by age were significantly different after 60 years, and CCT showed a statistically significant difference after 50 years. (Fig. 2).

The comparison of corneal parameters based on the duration of disease showed differences related to age group. ECD and CV after 60 years and CCT after 50 years in the group with a long duration of disease ( $\geq 10$ years) were significantly different from the control group. Hexagonality showed a significant difference only for ages between 60 and 69 years (Fig. 3). Between the long duration group ( $\geq 10$ years) and the short duration group ( $<10$ years), only ECD showed a statistically significant difference after age 60 (Fig. 3A).

In the high HbA1c group ( $\geq 7 \%$ ), the ECD at ages between 60 and 79 years were significantly lower than those of the control group ( $p=0.000$ and 0.002 , respectively), and CV was statistically different from that of the control group after 60 years of age. $(p<0.05)$ (Fig. 4A, B). There were differences in CCT at ages between 40 and 59 years $(p=0.017$ and 0.000 , respectively) and hexagonality at ages between 60 and 69 years $(p=0.025)$, however, there was no clear tendency related to aging (Fig. $4 C, D)$. In the low HbA1c group ( $<7 \%$ ), only CCT in the $50 \mathrm{~s}$ and $80 \mathrm{~s}$ and CV at ages between 60 and 79 years were significantly different from those of the control group (Fig. 4B, D). Only ECD in the high HbA1c ( $\geq 7 \%$ ) and low HbA1c $(<7 \%)$ groups showed a statistically significant difference at ages between 60 and 69 years $(p=0.001)$ (Fig. 4A).

\section{Pearson Correlation analysis}

Pearson correlation analysis showed that age had significant correlations with ECD, CV, hexagonality, and CCT. In addition, DM duration had significant correlations with ECD. (Table 3.) However, the HbA1c levels had no significant correlations with ECD, CV, hexagonality, and CCT. 
Table 3

Correlations between endothelial cell parameters and age, diabetic duration, and $\mathrm{HbA} 1 \mathrm{c} \%$ in the diabetic group

\begin{tabular}{|c|c|c|c|c|}
\hline & ECD & $\mathrm{CV}$ & Hexagonality & CCT \\
\hline \multicolumn{5}{|l|}{ Age } \\
\hline $\mathrm{R}$ value & -0.257 & 0.252 & -0.136 & -0.141 \\
\hline$P$ value & 0.000 & 0.000 & 0.000 & 0.000 \\
\hline \multicolumn{5}{|c|}{ DM duration } \\
\hline $\mathrm{R}$ value & -0.167 & 0.059 & -0.059 & 0.020 \\
\hline$P$ value & 0.000 & 0.057 & 0.060 & 0.535 \\
\hline \multicolumn{5}{|l|}{$\mathrm{HbA} 1 \mathrm{c}$} \\
\hline $\mathrm{R}$ value & 0.064 & -0.051 & -0.010 & 0.057 \\
\hline$P$ value & 0.058 & 0.130 & 0.765 & 0.096 \\
\hline
\end{tabular}

\section{Discussion}

DM affects all structural layers of the cornea, including the corneal epithelium, corneal nerves, tear film, and corneal endothelium. ${ }^{17}$ Clinically observed corneal diabetic complications include recurrent epithelial erosion, delayed wound repair, neurotrophic ulcers, and decreased corneal sensitivity. Morphological and functional changes also occur in the corneal endothelium, and diabetes carries an increased risk of endothelial complications after intraocular surgery. Although the pathophysiological mechanisms underlying diabetic damage of the corneal endothelium are not yet clear, osmotic damage due to excessive sorbitol accumulation, oxidative damage due to glycation end-product accumulation, and the reduction of corneal endothelial cell adhesion due to direct adhesive protein modifications of Descemet's membrane by AGEs has been proposed. ${ }^{18-20}$

Several studies have evaluated the endothelium in patients with type 2 diabetes and normal subjects. Most of them agree that the corneal endothelium of diabetic patients changes morphologically. ${ }^{10,18,21-23}$ Su et al. ${ }^{18}$ reported that diabetics had thicker corneas and decreased ECD than persons without diabetes. Roszkowska et al. ${ }^{23}$ reported a significant decrease in cell density in type 2 diabetic patients. Other studies also showed a significant decrease in ECD and hexagonality and an increase in CV in type 2 DM patients. ${ }^{12,14}$ These results are consistent with the findings of this study. However, several studies found no difference between the ECDs of diabetics and normal subjects. ${ }^{24,25}$ The effect of DM on the corneal endothelium remains controversial. 
In the present study, it was detected that ECD, CV, hexagonality, and CCT showed significant differences between long durations of diabetes ( $\geq 10$ years) and control in all age (Fig. 1) However, only CV and CCT showed significant differences between short durations of diabetes ( $<10$ years) and control. In addition, high $\mathrm{HbA1c}(\geq 7 \%)$ patients showed differences in ECD, CV, and CCT, and low HbA1c patients (<7\%) showed only differences in CV and CCT. Therefore, it can be assumed that the duration of DM or poor blood glucose control had a greater effect on corneal endothelial change. Previous studies also reported that changes in ECD, CV, and CCT are associated with the duration of DM, and patients who had had DM for more than 10 years had more significant changes. ${ }^{14,26}$ Corneal endothelial cell change in diabetes is a chronic microvascular complication, and complications occur more frequently when DM is prolonged and patients are chronically exposed to hyperglycemic conditions. ${ }^{27}$ On the other hand, $\mathrm{HbA} 1 \mathrm{c}$ reflects shortterm DM control, and it is reported that CCT may change within a short period depending on the HbA1c level. ${ }^{14}$ In our study, long-standing diabetic patients showed more significant differences in overall corneal parameters between DM and control groups than in high Hb1Ac patients. This suggests that the duration of diabetes is more strongly associated with chronic corneal endothelial damage than HbA1c. Therefore, the duration of diabetes is recommended over $\mathrm{HbA} 1 \mathrm{c}$ for predicting corneal endothelial cell damage.

According to the results of the correlation analysis, age was the most important factor, and it correlated with all corneal parameters, and DM duration correlated with ECD. On the other hand, HbA1c did not show significant correlations with the corneal parameters; This is because HbA1c reflects short-term diabetic control, and it does not reflect chronic corneal endothelial cell damage.

When the groups were stratified by age, ECD, hexagonality, and CCT decreased whereas CV tended to increase with aging, which was consistent with the findings of previous studies. ${ }^{28} \mathrm{~A}$ few papers have compared diabetic patients by dividing them into age groups. In 2012, Rachapalle et al. ${ }^{10}$ analyzed the data of 1191 DM patients and 121 non-diabetic patients stratified by age. They reported that CV and hexagonality were greater in diabetic patients than in controls aged between 50 and 69 years, and CCT was thicker in diabetic patients than in controls aged between 60 and 69 years. In this study, most corneal parameters showed significant differences in the DM and control groups after the age of 60 years. The reason for the pronounced difference between the DM and control groups as the age increased was not revealed. This is presumed to be because the DM duration is more likely to become longer as the elderly age, and the older person is vulnerable to corneal endothelial cell damage.

When the DM duration and $\mathrm{HbA} 1 \mathrm{c}$ of the DM and control groups stratified by age were analyzed, CCT showed a significant difference after 50 years in patients with long-standing diabetes ( $\geq 10$ years) and $E C D, C V$, and hexagonality showed significant differences after 60 years. Similar trends were observed in the high HbA1c group ( $\geq 7 \%$ ); CCT showed a difference at the age of 40 years, whereas ECD, CV, and hexagonality showed significant differences at the age of 60 . Although the overall corneal parameters were more affected by DM duration than $\mathrm{HbA1c}$, but $\mathrm{HbA1c}$ can affect CCT for a short period of time. Therefore, it is assumed that there was a difference between the two groups even at age 40. 
The limitation of this study is that the results were cross-sectional, and prospective changes were not confirmed. In future studies, it is necessary to observe consecutive changes of corneal parameters over time. Despite these limitations, the relatively large sample of more than 1000 patients subdivided into age groups in this study is an advantage.

In conclusion, the corneas of patients with type $2 \mathrm{DM}$ showed an increase in thickness and $\mathrm{CV}$ and reductions in ECD and hexagonality compared with the healthy controls. These changes were more in older type 2 DM patients ( $\geq 60$ years) with a long duration of disease ( $\geq 10$ years) and high HbA1c ( $\geq$ $7 \%$ ). In the correlation analysis, age and the duration of DM affected ECD. These results are from a large population-based sample, and which support the theory that type $2 \mathrm{DM}$ can affect corneal endothelial cells. Therefore, it is necessary to evaluate not only the retina but also the corneal endothelial cells regularly during follow-up for type $2 \mathrm{DM}$ patients.

\section{Methods}

\section{The study population}

This cross-sectional study complied with the Declaration of Helsinki, and it was approved by the Institutional Review Board of Kyung Hee University Hospital at Gangdong (approval number: 2020-10016). An exemption was granted from the requirement for informed consent by the Institutional Review Board of Kyung Hee University Hospital at Gangdong because the study had a retrospective design. Patients over 40 years old age, who visited our outpatient clinic between 2016 and 2019 and underwent specular microscopy were included. The diagnosis of type 2 diabetes and the duration of diabetes were analyzed based on medical history, and all the diabetic patients were taking oral or parenteral antidiabetic medication. In the DM group, the serum glycosylated hemoglobin ( $\mathrm{HbA1c}$ ) levels were analyzed within 2 months before and after ophthalmic visits. The patients underwent corneal specular microscopy, slitlamp examination, fundus examination, and corneal topography. The exclusion criteria included glaucoma, uveitis, intraocular surgery or laser treatment, previous use of contact lenses, and history of corneal diseases such as keratoconus, Fuchs endothelial dystrophy, and corneal opacities.

\section{Analysis of Corneal endothelial cells and central corneal thickness}

To assess the corneal endothelial status, the central ECD (cells $/ \mathrm{mm}^{2}$ ), variations in the size of endothelial cells (CV) (\%), and the percentage of hexagonal cells (\%) were analyzed using Topcon SP3000P non-contact endothelial specular microscope (Topcon Corporation, Tokyo, Japan). The subjects were asked to look at the central fixation target, and the auto-alignment function was used. The central endothelial cell density (cells $/ \mathrm{mm}^{2}$ ), CV, and the percentage of hexagonal cells were calculated using the software of the specular microscope. CCT was obtained using a non-contact Pentacam rotating Scheimpflug camera (Oculus, Wetzlar, Germany). During the measurements, the subjects fixated on a distant target. The internal software (Pentacam Basic software) automatically determined corneal thickness. 


\section{Statistical analysis}

The ECD, CV, hexagonlaity, and CCT of the diabetic patients and control subjects were compared using the independent samples $t$-test in SPSS version 18.0 software (SPSS Inc., Chicago, USA). A Pearson correlation test was performed to determine the relationships between corneal changes, DM duration, and $\mathrm{HbA} 1 \mathrm{c} \%$. A p-value of less than 0.05 was considered to be statistically significant.

\section{Declarations}

\section{Acknowledgements}

This research was supported by the National Research Foundation of Korea (NRF) funded by the Ministry of Science, ICT \& Future Planning (2018M3A9E8078812 and 2020R1F1A1072369). Funding was provided for English editing during manuscript preparation and for the publishing fee.

\section{Author contributions}

The authors were involved in the following aspects of the study: design and conduct (T.G.K., Y.J.K.); collection of the data (T.G.K); management (T.G.K., Y.J.K.); analysis (T.G.K.); interpretation (T.G.K., Y.J.K.); preparation of the article (T.G.K., Y.J.K.); review and approval of the manuscript (T.G.K.).

\section{Additional information}

The authors declare no competing interests.

\section{References}

1. Tuft, S.J. \& Coster, D.J. The corneal endothelium. Eye (Lond). 4, 389-424 (1990).

2. Wilson, R.S. \& Roper-Hall, M.J. Effect of age on the endothelial cell count in the normal eye. Br. J. Ophthalmol. 66, 513-515 (1982).

3. Mäkitie, J, Vannas A \& Koskenvuo M. Corneal endothelial cells in monoand di-zygotic twins. Invest. Ophthalmol. Vis. Sci. 24, 1029-1032 (1983).

4. Matsuda M, Yee RW \& Edelhauser HF. Comparison of the corneal endothelium in an American and a Japanese population. Arch. Ophthalmol. 103, 68-70 (1985).

5. Laing, R.A., Sanstrom, M.M., Berrospi, A.R. \& Leibowitz, H.M. Changes in the corneal endothelium as a function of age. Exp. Eye. Res. 22, 587-594 (1976).

6. Bourne, W.M., Nelson, L.R. \& Hodge, D.O. Continued endothelial cell loss ten years after lens implantation. Ophthalmology. 101, 1014-1022 (1994).

7. Slingsby, J.G. \& Forstot, S.L. Effect of blunt trauma on the corneal endothelium. Arch. Ophthalmol. 99, 1041-1043 (1981). 
8. Schultz, R.O., Glasser, D.B., Matsuda, M., Yee, R.W. \& Edelhauser, H.F. Response of the corneal endothelium to cataract surgery. Arch. Ophthalmol. 104, 1164-1169 (1986).

9. Vieira-Potter, V.J., Karamichos, D. \& Lee, D.J. Ocular complications of diabetes and therapeutic approaches. Biomed. Res. Int. 2016, 3801570; 10.1155/2016/3801570 (2016).

10. Sudhir, R.R., Raman, R. \& Sharma, T. Changes in the corneal endothelial cell density and morphology in patients with type 2 diabetes mellitus: a populationbased study, Sankara Nethralaya diabetic retinopathy and molecular genetics study (SN-DREAMS, report 23). Cornea. 31, 1119-1122 (2012).

11. Choo, M., Prakash, K., Samsudin, A., Soong, T., Ramli, N. \& Kadir, A. Corneal changes in type II diabetes mellitus in Malaysia. Int. J. Ophthalmol. 3, 234-236 (2010).

12. Inoue, K., Kato, S., Inoue, Y., Amano, S. \& Oshika, T. The corneal endothelium and thickness in type II diabetes mellitus. Jpn. J. Ophthalmol. 46, 65-69 (2002).

13. Schultz, R.O., Matsuda, M., Yee, R.W., Edelhauser, H.F. \& Schultz, K.J. Corneal endothelial changes in type I and type II diabetes mellitus. Am. J. Ophthalmol. 98, 401-410 (1984).

14. Lee, J.S., Oum, B.S., Choi, H.Y., Lee, J.E. \& Cho, B.M. Differences in corneal thickness and corneal endothelium related to duration in diabetes. Eye (Lond). 20, 315-318 (2006).

15. Storr-Paulsen, A., Singh, A., Jeppesen, H., Norregaard, J.C. \& Thulesen, J. Corneal endothelial morphology and central thickness in patients with type II diabetes mellitus. Acta. Ophthalmol. 92, 158-160 (2014).

16. Su, D.H. et al. Diabetes, hyperglycemia, and central corneal thickness: the Singapore Malay Eye Study. Ophthalmology. 115, 964-968 (2008).

17. Ljubimov, A.V. Diabetic complications in the cornea. Vision. Res. 139, 138-152 (2017).

18. Lutty, G.A. Effects of diabetes on the eye. Invest Ophthalmol Vis Sci. 54, 81-87 (2013).

19. Kaji, Y. et al. Advanced glycation end products in diabetic corneas. Invest. Ophthalmol. Vis. Sci.41, 362-368 (2000).

20. Kaji, Y. et al. Advanced glycation end products in Descemet's membrane and their effect on corneal endothelial cell. Curr. Eye. Res. 23, 469-77 (2001).

21. Agamy, A.E. \& Alsubaie, S. Corneal endothelium and central corneal thickness changes in type 2 diabetes mellitus. Clin. Ophthalmol. 11, 481-486 (2017).

22. Galgauskas, S., Laurinaviciute, G., Norvydaite, D., Stech, S. \& Asoklis, R. Changes in Choroidal Thickness and Corneal Parameters in Diabetic Eyes. Eur. J. Ophthalmol. 26, 163-167 (2015).

23. Roszkowska, A.M., Tringali, C.G., Colosi, P., Squeri, C.A. \& Ferreri, G. Corneal endothelium evaluation in type I and type II diabetes mellitus. Ophthalmologica. 213, 258-261 (1999).

24. Inoue, K. et al. Corneal endothelial cell morphology in patients undergoing cataract surgery. Cornea. 21, 360-363 (2002).

25. Hugod, M. et al. Corneal endothelial cell changes associated with cataract surgery in patients with type II diabetes mellitus. Cornea. 30, 749-753 (2011). 
26. Briggs, S., Osuagwu, U.L. \& AlHarthi, E.M. Manifestations of type 2 diabetes in corneal endothelial cell density, corneal thickness and intraocular pressure. J. Biomed. Res. 30, 46-51 (2016).

27. Cade, W.T. Diabetes-Related Microvascular and Macrovascular Diseases in the Physical Therapy Setting. Phys. Ther. 88, 1322-1335 (2008).

28. Vitályos, G. et al. Effects of aging on corneal parameters measured with Pentacam in healthy subjects. Sci. Rep. 9, 3419; 10.1038/s41598-019-39234-x (2019).

\section{Figures}
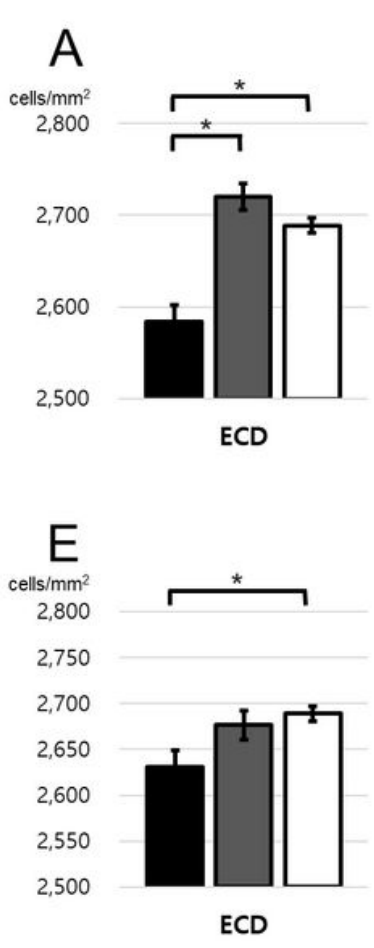

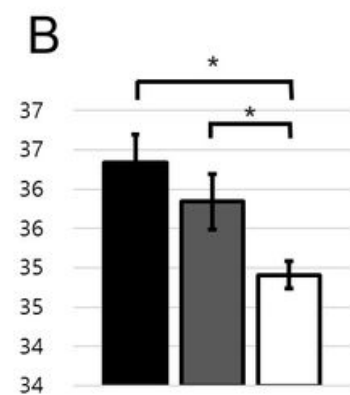

$\mathrm{CV}$

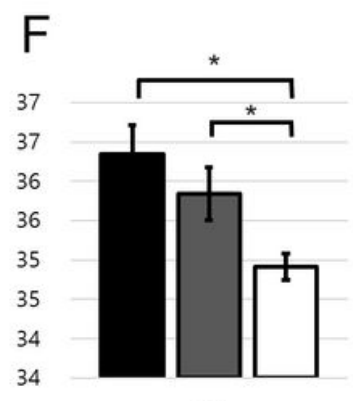

$\mathrm{cV}$

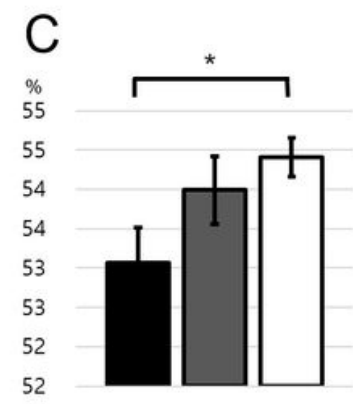

Hexagonality

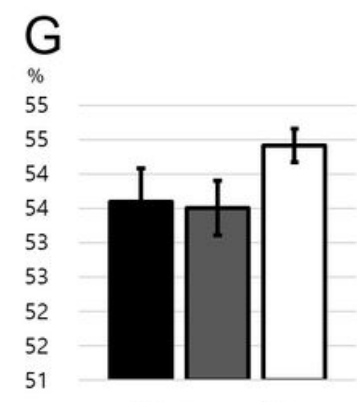

Hexagonality

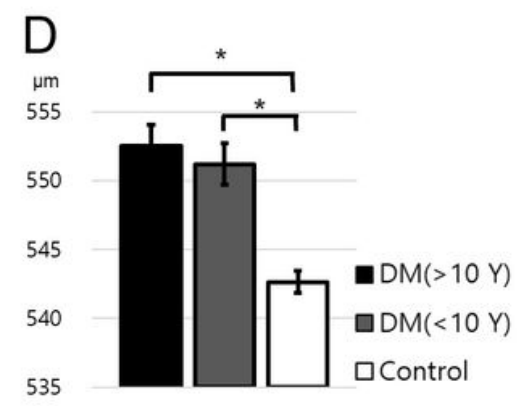

CCT

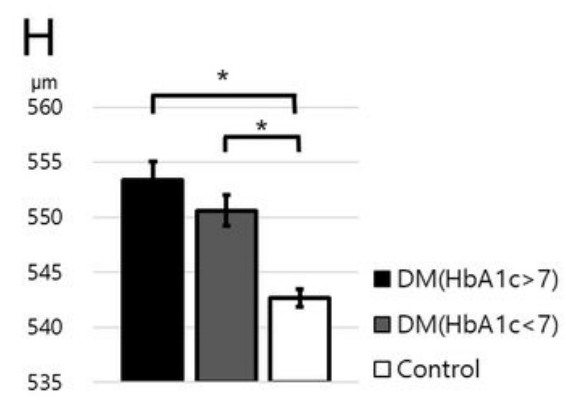

cCT

${ }^{*} \mathrm{p}<0.05$, by independent $\mathrm{t}$-test

Figure 1

Comparison of endothelial cell parameters of diabetic patients and controls based on diabetic duration and $\mathrm{HbA} 1 \mathrm{c} \%$ across all ages. (A-D) Corneal endothelial cell parameters based on disease duration. (E-H) Corneal endothelial cell parameters based on HbA1c concentration. ECD: Endothelial Cell Density; CV: Coefficient of Variation; CCT: Central Corneal Thickness 

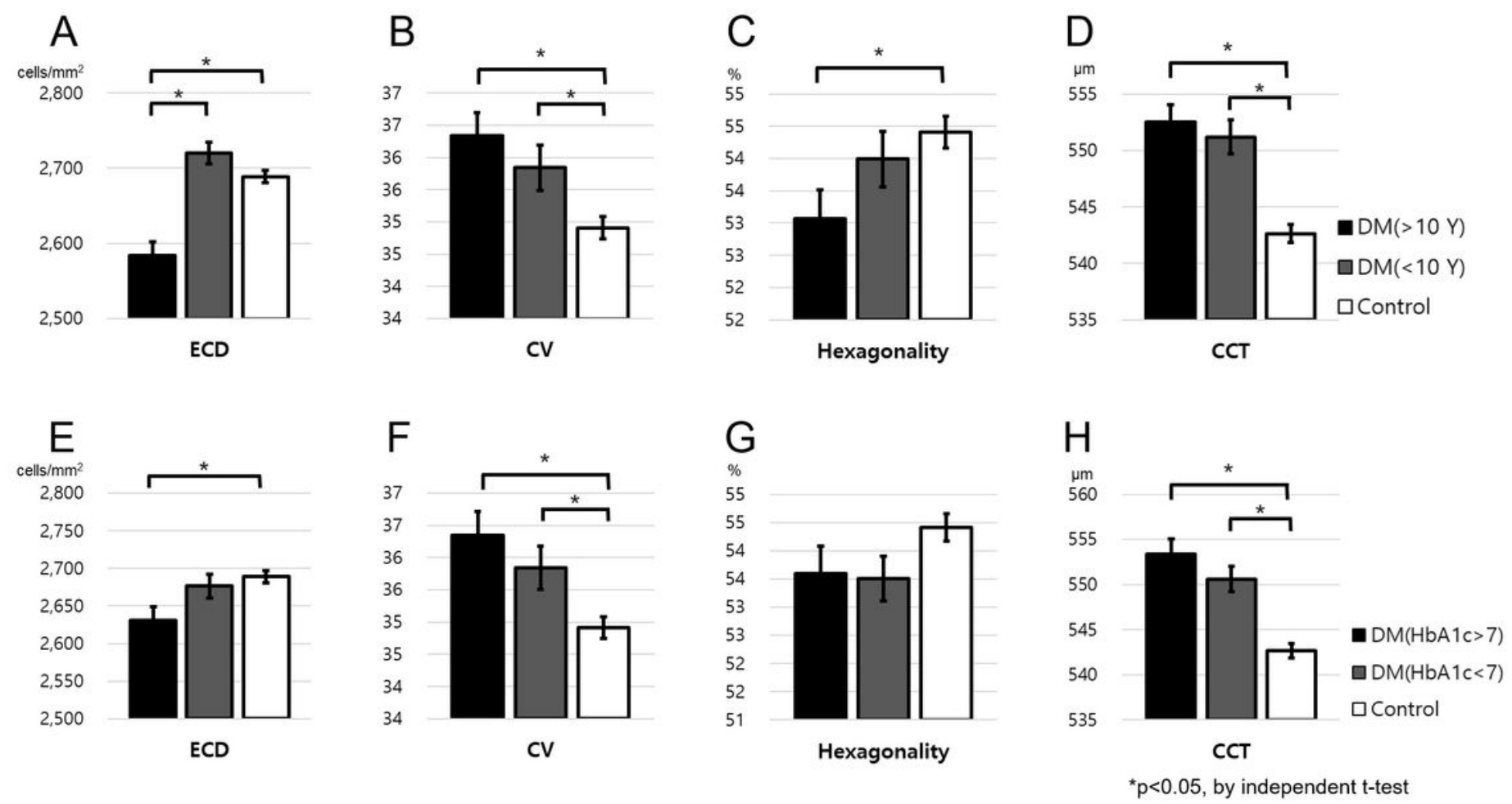

Figure 1

Comparison of endothelial cell parameters of diabetic patients and controls based on diabetic duration and $\mathrm{HbA} 1 \mathrm{c} \%$ across all ages. (A-D) Corneal endothelial cell parameters based on disease duration. (E-H) Corneal endothelial cell parameters based on $\mathrm{HbA1c}$ concentration. ECD: Endothelial Cell Density; CV: Coefficient of Variation; CCT: Central Corneal Thickness 

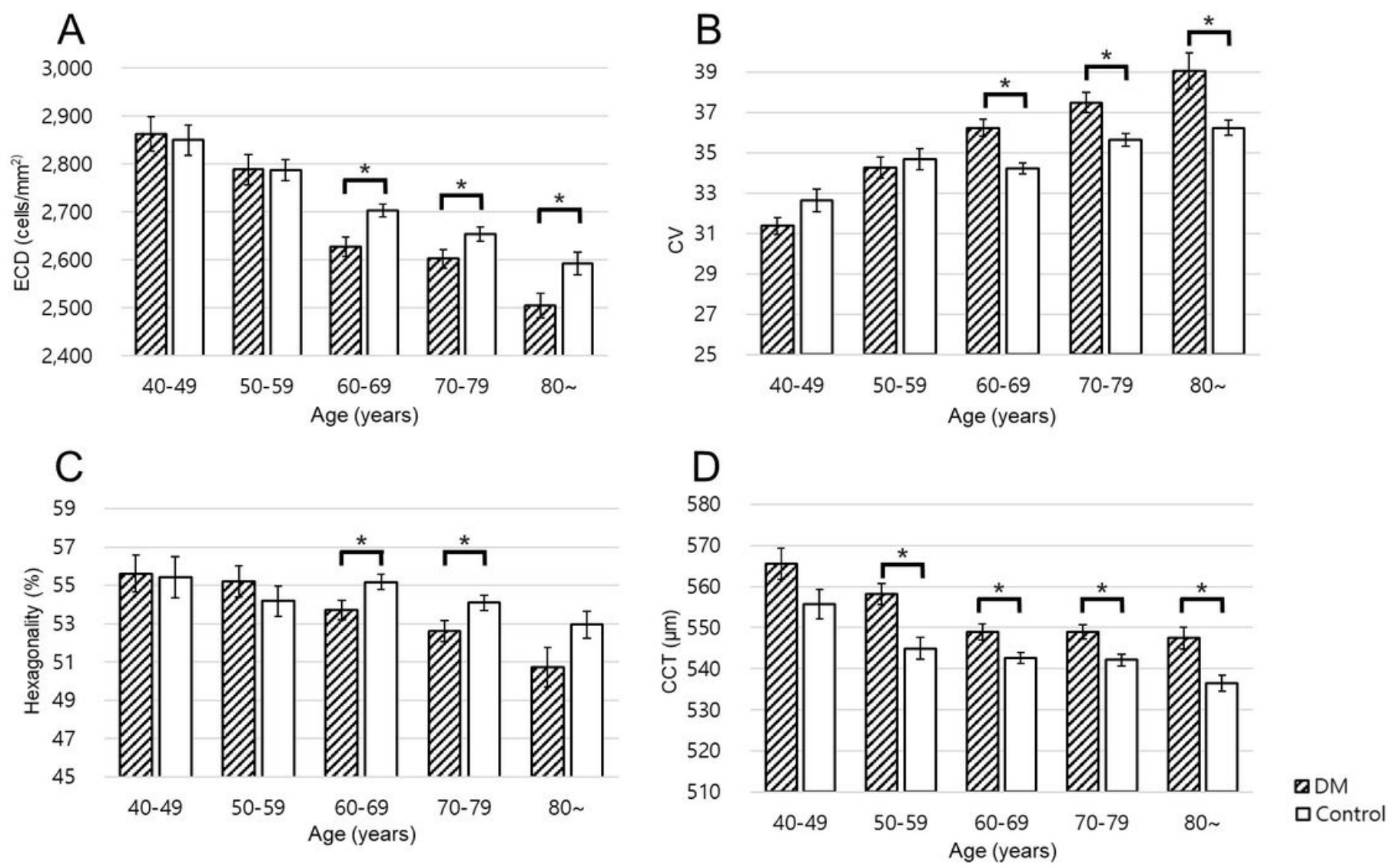

${ }^{*} \mathrm{p}<0.05$, by independent $\mathrm{t}$-test

Figure 2

Comparison of endothelial cell parameters of diabetic patients and control based on age. (A) Mean ECD, (B) mean CV, (C) mean hexagonality and (D) mean CCT across the age groups. ECD: Endothelial Cell Density; CV: Coefficient of Variation; CCT: Central Corneal Thickness 

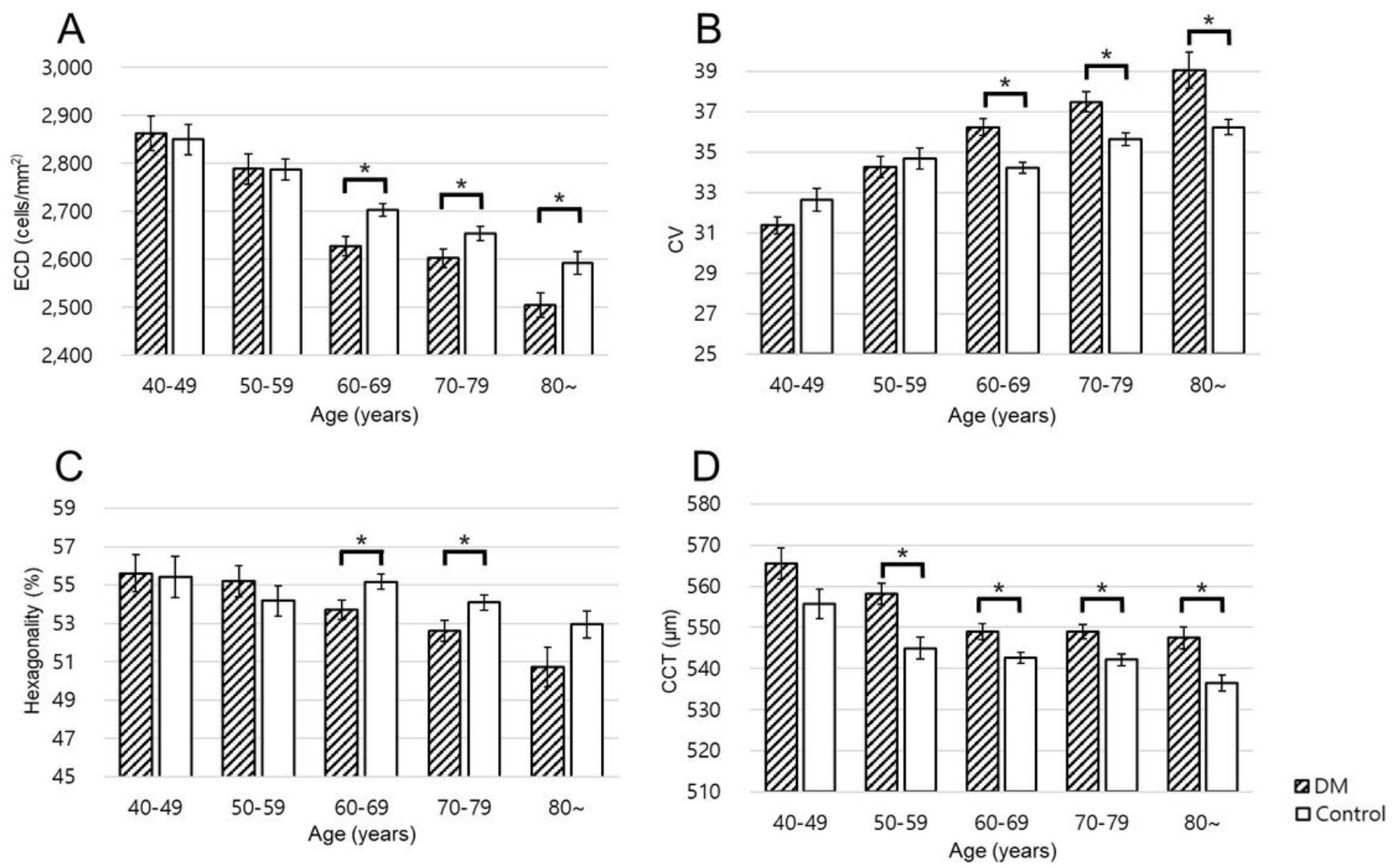

${ }^{*} \mathrm{p}<0.05$, by independent t-test

Figure 2

Comparison of endothelial cell parameters of diabetic patients and control based on age. (A) Mean ECD, (B) mean CV, (C) mean hexagonality and (D) mean CCT across the age groups. ECD: Endothelial Cell Density; CV: Coefficient of Variation; CCT: Central Corneal Thickness 

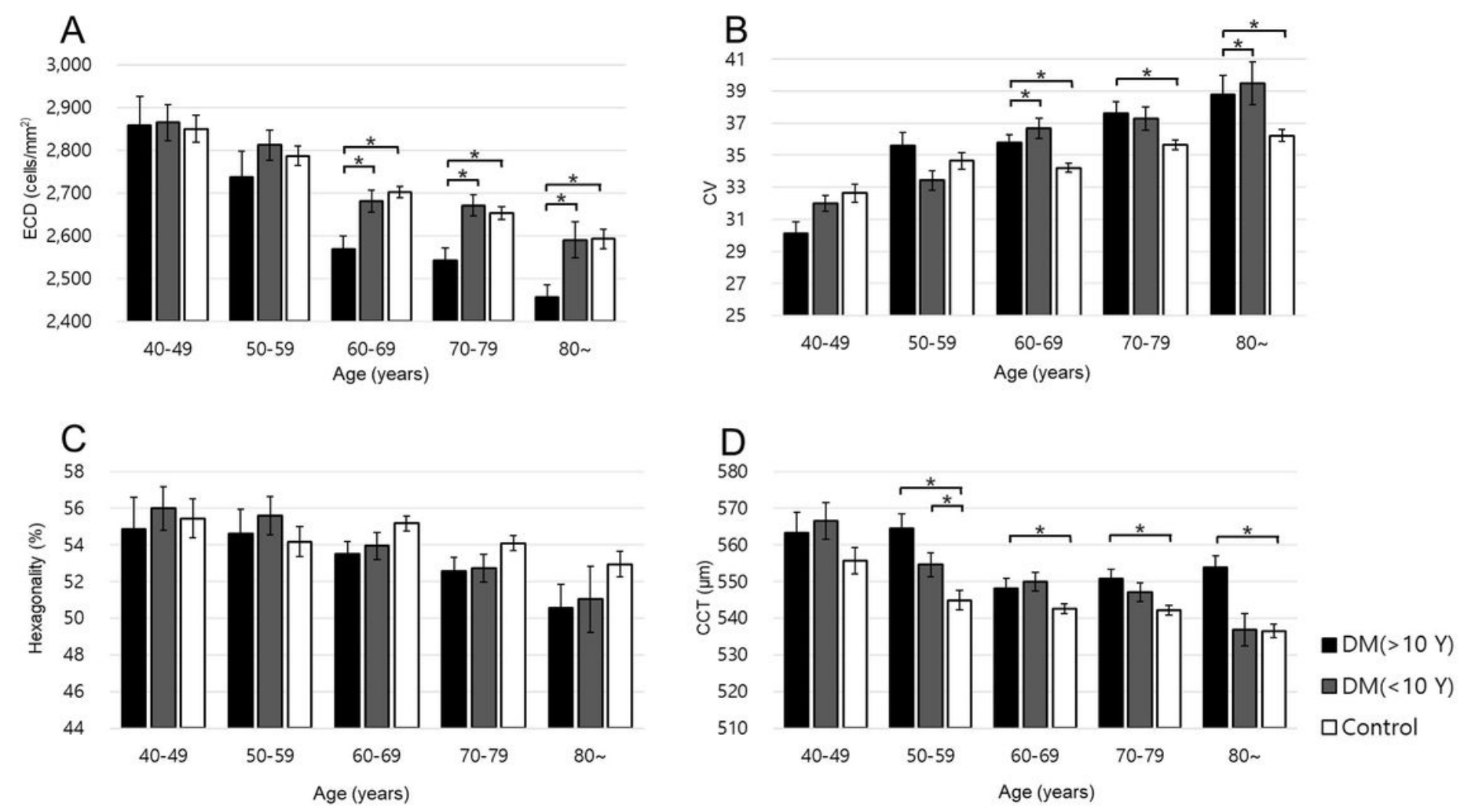

${ }^{*} p<0.05$, by independent t-test

\section{Figure 3}

Comparison of endothelial cell parameters of diabetic patients and control based on age. (A) Mean ECD, (B) mean CV, (C) mean hexagonality and (D) mean CCT across the age groups. ECD: Endothelial Cell Density; CV: Coefficient of Variation; CCT: Central Corneal Thickness 

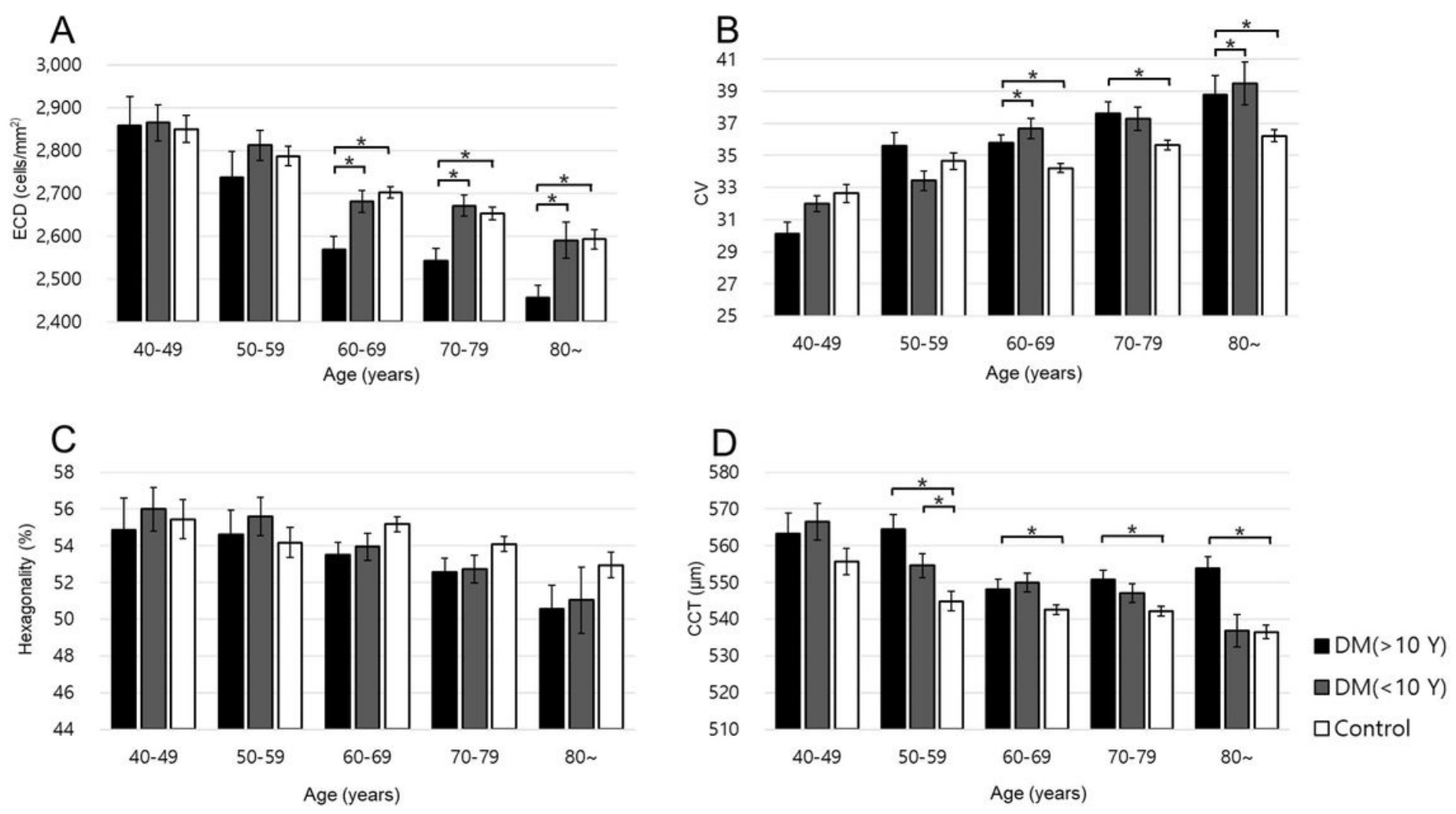

${ }^{*} p<0.05$, by independent t-test

\section{Figure 3}

Comparison of endothelial cell parameters of diabetic patients and control based on age. (A) Mean ECD, (B) mean CV, (C) mean hexagonality and (D) mean CCT across the age groups. ECD: Endothelial Cell Density; CV: Coefficient of Variation; CCT: Central Corneal Thickness 

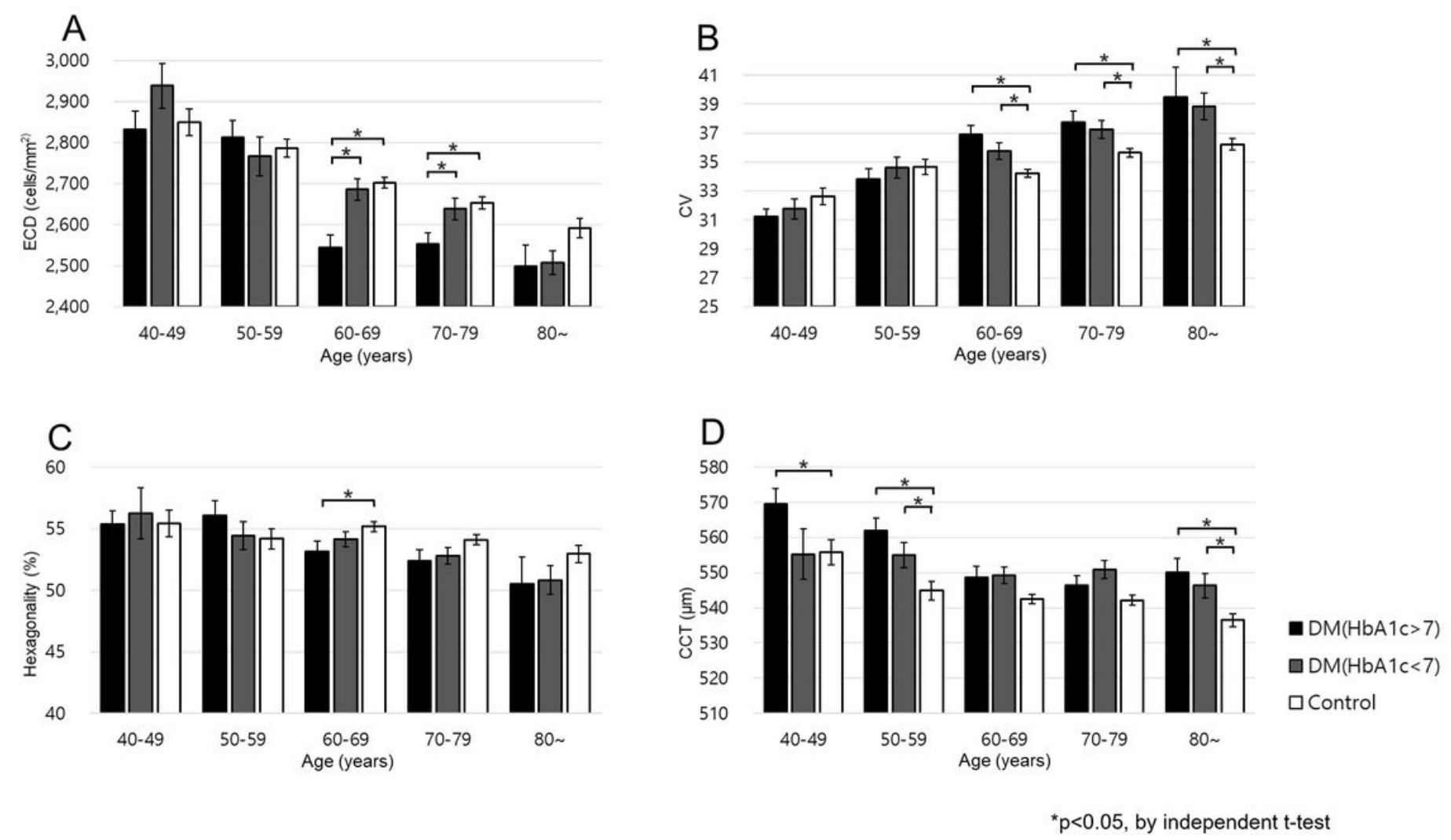

Figure 4

Comparisons of the mean values of ECD (A) , CV (B), hexagonality (C) and CCT (D) of the diabetic groups based on $\mathrm{HbA1c} \%$ across all age groups. ECD: Endothelial Cell Density; CV: Coefficient of Variation; CCT: Central Corneal Thickness 

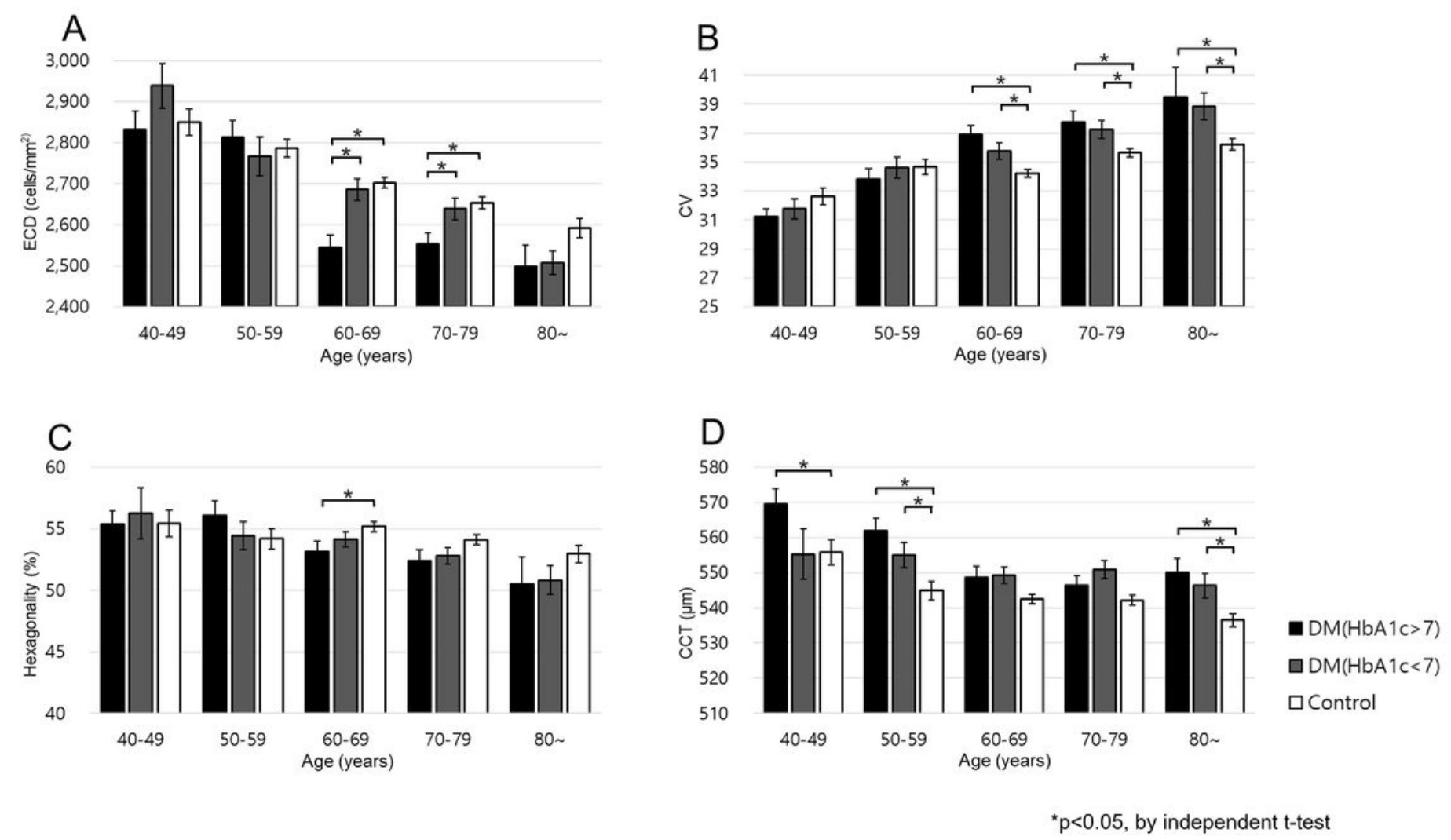

Figure 4

Comparisons of the mean values of ECD (A) , CV (B), hexagonality (C) and CCT (D) of the diabetic groups based on $\mathrm{HbA1c} \%$ across all age groups. ECD: Endothelial Cell Density; CV: Coefficient of Variation; CCT: Central Corneal Thickness 